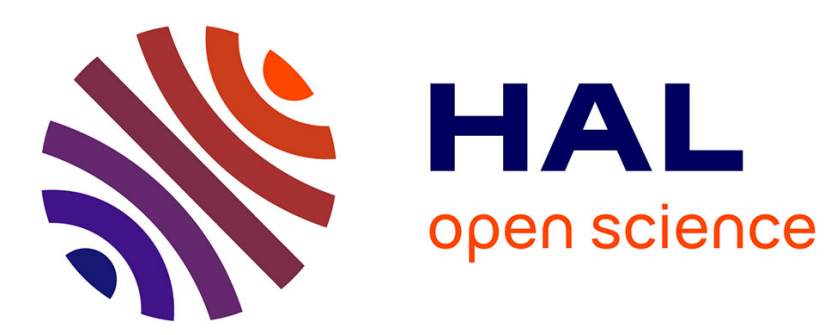

\title{
On the Maximal Subgroup of Automorphisms of a Free Group FN which Fix a Point of the Boundary FN
}

\author{
Arnaud Hilion
}

\section{To cite this version:}

Arnaud Hilion. On the Maximal Subgroup of Automorphisms of a Free Group FN which Fix a Point of the Boundary FN. International Mathematics Research Notices, 2007, 10.1093/imrn/rnm066 . hal-01218379

\section{HAL Id: hal-01218379 \\ https://hal.science/hal-01218379}

Submitted on 21 Oct 2015

HAL is a multi-disciplinary open access archive for the deposit and dissemination of scientific research documents, whether they are published or not. The documents may come from teaching and research institutions in France or abroad, or from public or private research centers.
L'archive ouverte pluridisciplinaire HAL, est destinée au dépôt et à la diffusion de documents scientifiques de niveau recherche, publiés ou non, émanant des établissements d'enseignement et de recherche français ou étrangers, des laboratoires publics ou privés. 


\title{
ON THE MAXIMAL SUBGROUP OF AUTOMORPHISMS OF A FREE GROUP $F_{N}$ WHICH FIX A POINT OF THE BOUNDARY $\partial F_{N}$
}

\author{
ARNAUD HILION
}

\begin{abstract}
We prove that the stabilizer of an attractive fixed point in $\partial F_{N}$ of an irreducible with irreducible powers automorphism of a free group $F_{N}$ is virtually cyclic. The proof uses the attractive lamination of such an automorphism. We also establish the complete list of all possible maximal subgroups of $\operatorname{Aut}\left(F_{2}\right)$ which fix a point of the boundary $\partial F_{2}$.
\end{abstract}

\section{INTRODUCTION}

Let $F_{N}$ denote the free group of finite rank $N \geqslant 2$. The boundary $\partial F_{N}$ of $F_{N}$, a Cantor set, can be viewed as the set of right-infinite reduced words (in some fixed basis of $F_{N}$ ). An automorphism $\varphi$ of a free group $F_{N}$ induces canonically a homeomorphism $\partial \varphi$ of the boundary $\partial F_{N}$. For instance, the conjugation $i_{u}$ by $u \in F_{N}$ (given by $i_{u}(g)=u g u^{-1}$ for all $g \in F_{N}$ ) induces the left translation $\partial i_{u}: \partial F_{N} \rightarrow \partial F_{N}$, given by $X \mapsto u X$, on the boundary.

It has been proved by G. Levitt and M. Lustig in [16] that, for all automorphisms $\varphi$ of $F_{N}$, the induced homeomorphism $\partial \varphi$ has always at least two periodic points in $\partial F_{N}$. It is quite natural to pose the inverse problem: if $X$ is a point of $\partial F_{N}$, for which automorphisms $\varphi \in \operatorname{Aut}\left(F_{N}\right)$, does the induced homeomorphism $\partial \varphi$ of $\partial F_{N}$ fix $X$ ? We denote by $\operatorname{Stab}(X)$ the stabilizer of $X$ : it is the subgroup of all automorphisms $\varphi \in \operatorname{Aut}\left(F_{N}\right)$ such that $\partial \varphi(X)=X$. In this article, we focus on the following question: which subgroups of $A u t\left(F_{N}\right)$ can be obtained as stabilizers of a point in the boundary of $F_{N}$, up to isomorphism?

An automorphism $\varphi \in \operatorname{Aut}\left(F_{N}\right)$ is said iwip (i.e. irreducible, with irreducible powers) if no non-trivial free factor of $F_{N}$ is mapped by some power $\varphi^{k}(k \geqslant 1)$ to a conjugate of itself. We prove:

Theorem I. If $X \in \partial F_{N}$ is an attractive fixed point of an iwip automorphism, then $\operatorname{Stab}(X)$ is infinite cyclic.

The study of automorphisms of free groups has been deeply influenced by Nielsen-Thurston theory of surface homeomorphisms, since any homeomorphism of a surface induces an automorphism on the fundamental group of this surface, which is a free group if the surface has at least one boundary component. It appears that iwip automorphisms of free groups are the natural equivalent of pseudo-Anosov homeomorphisms of surfaces. In order to highlight the main difficulty in the proof

Date: October 4, 2006.

Key words and phrases. automorphisms of free groups, iwip automorphism, attractive lamination, train-track, Dehn twist. 
of Theorem I, let us briefly discuss the analogous task for a pseudo-Anosov homeomorphism.

Let $\Sigma$ be a closed orientable surface of negative Euler characteristic. Its universal cover can be identified with the Poincaré disk $D$. Consider a pseudoAnosov homeomorphism $f_{0}$ of $\Sigma$ : the map $f_{0}$ fixes two transverse singular measured foliations $\mathcal{F}_{u}$ and $\mathcal{F}_{s}$ of $\Sigma$ (see [23], [11], [7]). These foliations can be lifted to two foliations $\tilde{\mathcal{F}}_{u}$ and $\tilde{\mathcal{F}}_{s}$ of $D$. A lift $f$ of $f_{0}$ to $D$ leaves $\tilde{\mathcal{F}}_{u}$ and $\tilde{\mathcal{F}}_{s}$ invariant and induces a homeomorphism $\partial f$ on the boundary $S_{\infty}$ of $D$ (the "circle at infinity"). If $f$ fixes a point $p \in D$, then $\partial f$ (or a positive power of $\partial f$ ) fixes the points of $S_{\infty}$ which are the ends of those (possibly singular) leaves of the foliations $\tilde{\mathcal{F}}_{u}$ and $\tilde{\mathcal{F}}_{s}$ which contain $p$. However, the generic situation is that $f$ does not fix any point of $D$ (see [15]). In such a case, $\partial f$ (or $\partial f^{2}$ ) has North-South dynamics: in particular, it has two fixed points, one attractive, and the other one repulsive. But these two fixed points can not be characterized as ends of invariant leaves in any one of the foliations $\tilde{\mathcal{F}}_{u}$ or $\tilde{\mathcal{F}}_{s}$

Now consider a point $X \in S_{\infty}$ which is fixed by $\partial f$, where $f$ a lift of a pseudo-Anosov $f_{0}$ of $\Sigma$. The given problem is to determine all homeomorphisms $g_{0}$ of $\Sigma$ which admit a lift $g$ such that $\partial g$ fixes $X$. In the special case where $X$ is an end of a leaf of $\tilde{\mathcal{F}}_{u}$ or $\tilde{\mathcal{F}}_{s}$, it is not too hard to convince ourselves that $g_{0}$ and $f_{0}$ must be powers of a same pseudo-Anosov homeomorphism, because the stabilizer of $\tilde{\mathcal{F}}_{u}$ (and $\tilde{\mathcal{F}}_{s}$ ) is virtually cyclic. But in the general case, where $f_{0}$ has no fixed point in $D$, this is far less evident. Theorem I settles this problem in the setting of an iwip automorphism of a free group.

In fact, the proof of Theorem I relies on earlier work on iwip automorphisms by M. Bestvina, M. Feighn and M. Handel [2]. An iwip automorphism can be profitably represented by a train-track map [4]: this is a homotopy equivalence of a graph $G$, with the remarkable property that no backtracking subpath occurs if one iterates the train-track map on any edge of $G$. The train-track map allows one to construct the attrative lamination of this iwip automorphism, which can be viewed as a minimal symbolic dynamical system (that is a minimal shift-invariant set of biinfinite reduced edge paths in $G$ ).

The main tool of this paper consists of giving a good representation of a given attractive fixed point $X$ of an iwip automorphism (cf proposition 4.1), which matches the language of the attractive lamination. Then one can relate the stabiliser of $X$ to the stabiliser of the attractive lamination (see Theorem 4.2), and later conclude using the fact proved in [2] that the stabilizer of the attractive lamination of an iwip automorphism is virtually cyclic.

The study of the combinatorial and dynamical properties of the attractive lamination and the fixed points of an iwip automorphism (and their relationships), which is the main ingredient in the proof of Theorem I, seems to be very promising by itself. For instance, it is used in [1] to construct, under additional algebraic conditions, a nice fractal representation for the automorphism. For general automorphisms, the study of the relationships between the combinatorics and dynamics, of attractive fixed points on one hand and attractive laminations in the other, is much more difficult; in the forthcoming paper [13] we will derive some results concerning the combinatorial complexity of such objects.

In the case of automorphisms of the free group of rank 2, we can give a more precise statement than that of Theorem I. This is essentially due to the 
fact that every automorphism of $F_{2}$ can be realized as a homeomorphism of a punctured torus $T$ (see [19]). There are three types of homeomorphisms of $T$ : periodic homeomorphisms, pseudo-Anosov homeomorphisms, and Dehn twists (and their roots). Combining these geometric data with the previous results on the stabilizer of the attractive fixed points of iwip automorphisms enable us to deduce Theorem II. Here a point of $\partial F_{N}$ is called rational if it is fixed by a conjugation, non-rational otherwise.

\section{Theorem II.}

(a) The stabilizer in Aut $\left(F_{2}\right)$ of a non-rational point in $\partial F_{2}$ is either trivial or infinite cyclic.

(b) The stabilizer in Aut $\left(F_{2}\right)$ of any rational point in $\partial F_{2}$ is isomorphic to one of the following groups: $\{1\}, \mathbb{Z}, \mathbb{Z}^{2},\left\langle x, y \mid x^{2}=y^{2}\right\rangle,\left\langle x, y \mid x^{2}=1,\left[x, y^{2}\right]=1\right\rangle$, or $\langle x, y| x y x=y x y>$.

The stabilizer of a rational point $X$ coincides with the stabilizer of the root $\sqrt{X} \in F_{N}$ of $X$. Thus the second part of Theorem II reduces to the problem of determining the stabilizer of an element of $F_{2}$, which has been solved in [5].

The solution to the general problem of determining the stabilizer of a point in $\partial F_{N}(N \geqslant 3)$ seems to us out of reach for the moment. However, we hope that we can obtain partial results using the combinatorial complexity of a point $X \in \partial F_{N}$, which seems to be deeply related to the geometry of the automorphims which fix $X$ (see [13]).

The outline of the paper is as follows. In section 1 we recall some basic results, and in particular the case of rational points is treated. The definition and several useful properties of train-track maps are recalled in section 2. Section 3 is concerned with laminations. Theorem I is proved in section 4, and Theorem II in section 5 .

Acknowledgements - This work is part of my PhD. I wish to thank my supervisor G. Levitt for the interest he has shown to it and the advices he has given to me. M. Lustig has warmly welcome me in Marseille where I benefit from his enthousiastic support.

\section{BASIC RESUltS}

1.1. Boundary of $F_{N}$ and boundary map induced by an automorphism. Let $F_{N}$ the free group of finite rank $N \geqslant 2$. We denote by $\partial F_{N}$ the (Gromov) boundary of $F_{N}$ (which can be alternatively viewed as the space of ends of $F_{N}$ ): it is a Cantor set, which compactifies $F_{N}$ to give $\bar{F}_{N}=F_{N} \cup \partial F_{N}$. If a basis of $F_{N}$ is fixed, one can consider $\partial F_{N}$ as the set of right infinite reduced words (in this basis).

Since an automorphism $\varphi \in \operatorname{Aut}\left(F_{N}\right)$ is a quasi-isometry of $F_{N}$ (for the word metric associated to some basis), it induces a homeomorphism $\partial \varphi$ of the boundary $\partial F_{N}$, and also a homeomorphism $\bar{\varphi}=\varphi \cup \partial \varphi$ of $\bar{F}_{N}$.

1.2. Rational and non-rational points. Consider $g \in F_{N}$. An element $u \in F_{N}$ is a root of $g$ if there exists $k \in \mathbb{N}, k \neq 0$, such that $g=u^{k}$. Among the roots of $g$, there is a single one for which $k$ is maximal: it is the root of $g$, denoted by $\sqrt{g}$. Remark that $\sqrt{g}$ can also be defined as the shortest root of $g$ (for the length of words in any fixed basis of $F_{N}$ ). 
Consider $g \in F_{N}, g \neq 1$. Then sequence $g^{k}$ converges towards a point in $\partial F_{N}$, as $k \rightarrow \infty$, denoted by $g^{\infty}$ : such a point is called a rational point and we say that $g$ is a root of this point. One can check that if $g, g^{\prime} \in F_{N}$ are roots of $X$, then $\sqrt{g}=\sqrt{g^{\prime}}$, and thus we can define the root of $X$, denoted by $\sqrt{X}$, as the root of $g$. We define $g^{-\infty}$ as $\left(g^{-1}\right)^{\infty}$.

Proposition 1.1. If $X \in \partial F_{N}$ is a rational point, then the stabilizer of $X$ in Aut $\left(F_{N}\right)$ is equal to the stabilizer of $\sqrt{X}$.

Proof. Let's denote $x=\sqrt{X}$. It is clear that if $x \in \operatorname{Fix} \varphi$, then $\partial \varphi$ fixes $X=x^{+\infty}$. Conversely, if $\partial \varphi$ fixes $X=x^{+\infty}$, then $x^{+\infty}=\varphi(x)^{+\infty}$. Hence $x=\sqrt{\varphi(x)^{+\infty}}$, i.e. there exists $p>0$ such that $\varphi(x)=x^{p}$. Since $\varphi$ is an automorphism, necessarily one has $p=1$, and thus $\varphi(x)=x$.

Remark 1.1. It is proved in [18] that the stabilizer in $\operatorname{Aut}\left(F_{N}\right)$ of an element of $F_{N}$ is finitely presented. Hence the stabilizer of a rational point in $\partial F_{N}$ is finitely presented.

If $u \in F_{N}$, we denote by $i_{u}$ the inner automorphism given by: $i_{u}(g)=u g u^{-1}$, for any $g \in F_{N}$. If $u \neq 1$, then $\partial i_{u}$ fixes exactly 2 points of $\partial F_{N}: u^{\infty}$ and $u^{-\infty}$. We note that one can alternatively define the rational points of $\partial F_{N}$ as the fixed points of any non-trivial inner automorphism.

Consider $\operatorname{Inn}\left(F_{N}\right)$, the group of inner automorphisms of $F_{N}$ : it is a normal subgroup of $\operatorname{Aut}\left(F_{N}\right)$, and the quotient group $\operatorname{Out}\left(F_{N}\right)=\operatorname{Aut}\left(F_{N}\right) / \operatorname{Inn}\left(F_{N}\right)$ is called the group of outer automorphisms of $F_{N}$.

Proposition 1.2. If $X \in \partial F_{N}$ is not a rational point, then the restriction of the quotient map Aut $\left(F_{N}\right) \rightarrow \operatorname{Out}\left(F_{N}\right)$ to $\operatorname{Stab}(X)$ is injective.

Proof. Assume $\varphi \in \operatorname{Aut}\left(F_{N}\right)$ and $u \in F_{N}, u \neq 1$. If $X$ is a fixed point of $\partial \varphi$ and of $\partial\left(i_{u} \circ \varphi\right)$, then $X$ is also a fixed point of $\partial i_{u}$, and hence a rational point: $X=u^{\infty}$ or $X=u^{-\infty}$.

1.3. Regular and singular fixed points of $\partial \varphi$. Let us denote by Fix $\varphi$ the fixed subgroup of $\varphi: \operatorname{Fix} \varphi=\left\{g \in F_{N}: \varphi(g)=g\right\}$. Since Fix $\varphi$ has finite rank (in fact, $\operatorname{rk}(\operatorname{Fix} \varphi) \leqslant N$, see [4]), its boundary $\partial \operatorname{Fix} \varphi$ embeds into $\partial F_{N}$ : it is a subset of the set Fix $\partial \varphi \subseteq \partial F_{N}$ of fixed points of $\partial \varphi$. Following J. Nielsen [20] [21] [22], a fixed point of $\partial \varphi$ is said to be singular if it is in $\partial \mathrm{Fix} \varphi$, to be regular otherwise.

A fixed point $X$ of $\partial \varphi$ is said to be attractive if there exists a neighbourhood $U$ of $X$ in $\bar{F}_{N}$ such that the sequences $\bar{\varphi}^{k}(x)$ converge to $X$ for all $x$ in $U$. A fixed point $X$ of $\partial \varphi$ is said to be repulsive if it is attractive for $\partial \varphi^{-1}$. The following lemma has been proved in [12].

Lemma 1.3. Let $\varphi \in A u t\left(F_{N}\right)$. A fixed point of $\partial \varphi$ is

- either singular,

- or attractive,

- or repulsive.

Example 1.1. Consider $u \in F_{N}, u \neq 1$, and the inner automorphism $i_{u}$. Then Fix $i_{u}$ is the infinite cyclic group generated by $\sqrt{u}$, and Fix $\partial i_{u}=\partial \mathrm{Fix} i_{u}$. Thus all the fixed points of $\partial i_{u}$ are singular. However, for all $X \in \partial F_{N} \backslash\left\{u^{-\infty}\right\}$, the sequence $\partial \varphi^{k}(X)$ converges to $u^{\infty}$. 


\section{TOPOLOGICAL REPRESENTATIVES - TRAIN-TRACKS}

I recall some definitions and facts, most of which can be found in section 2 of [3]. In particular, the graphs we are considering are in fact one-dimensional $\mathrm{CW}$-complexes.

\subsection{Marked graphs.}

2.1.1. Definition. The rose with $N$ petals, denoted $R_{N}$, is the graph with $N$ edges and one vertex $*$. One identifies $\pi_{1}\left(R_{N}, *\right)$ with $F_{N}$. A marked graph is a graph $G$ with a homotopy equivalence $\tau: R_{N} \rightarrow G$, called the marking. The marking induces an isomorphism which allows us to identify $\pi_{1}\left(R_{N}, *\right)$ with $F_{N}=\pi_{1}(G, \tau(*))$.

Let's denote by $\tilde{G}$ the universal cover of $G$, and by $p r: \tilde{G} \rightarrow G$ the natural projection: $\tilde{G}$ is a tree, whose vertices have finite valence. There is a natural action of $F_{N}$ on $\tilde{G}$ by deck transformations.

2.1.2. Paths. Following [3], a path of $\tilde{G}$ is an embedding $w: I \rightarrow X$, where $I$ is a closed real interval (i.e. $I=[a, b],[a,+\infty[]-,\infty, b]$ or $\mathbb{R}, a, b \in \mathbb{R}$ ), or possibly some constant map $[a, b] \rightarrow X$ (in this case, the path is said trivial): $w(a)$ (resp. $w(b))$ is the initial (resp. terminal) point of $w$. In fact, one will be only interested in the oriented image of $w$ that we also denote by $w ; w^{-1}$ is the path $w$ with the inverse orientation. If $v_{1}, v_{2}$ are points in $\tilde{G},\left[v_{1}, v_{2}\right]$ is the path whose initial point is $v_{1}$ and the terminal point is $v_{2}$. When the initial and terminal points of a path are vertices, one says that this path is an edge path.

A metric on $\tilde{G}$ is given by declaring that all the edges are isometric to the segment $[0,1]$. A path isometric to some bounded interval is called a finite path. A path in $\tilde{G}$ is called a ray (or a line) if it is isometric to $[0,+\infty[$ (or to $\mathbb{R}$ ). Finally, a path (or a finite path, a ray, a line) in $G$ is the composition of a path of $\tilde{G}$ by the projection $p r$.

2.1.3. Tightening. Remark that every continuous map $w:[a, b] \rightarrow \tilde{G}$ is homotopic relative endpoints to a unique finite path (which is $[w(a), w(b)])$. We denote this path by $[w]$, and we say that $[w]$ is obtained by tightening $w$. Similarly, every continuous map $w:[a, b] \rightarrow G$ is homotopic relative endpoints to a unique finite path, also denoted by $[w]$.

2.1.4. Boundary of the universal cover of a marked graph. Since $\tilde{G}$ is a simplicial tree whose vertices have finite valence, one can consider its (Gromov) boundary $\partial \tilde{G}$ : it is a Cantor set which compactifies $\tilde{G}$. If we fix a base point in $\tilde{G}$, using the marking $\tau: R_{N} \rightarrow G$ (or equivalentely the action of $F_{N}$ on $\tilde{G}$ ), $\partial \tilde{G}$ is naturally identified with $\partial F_{N}$.

Two rays of $\tilde{G}$ are equivalent if their intersection is a ray. Then $\partial \tilde{G}$ can be viewed as the set of equivalence classes of rays in $\tilde{G}$. In particular, a line in $\tilde{G}$ defines two distinct points of $\partial \tilde{G}$ (the equivalence classes of the rays obtained by cutting the line at a point), thus two distincts points of $\partial F_{N}$. Conversely, two distinct points of $\partial F_{N}$ define a unique line in $\tilde{G}$.

\subsection{Topological representatives.}


2.2.1. Definition. If $G$ is a marked graph (as in 2.1.1), a homotopy equivalence $f_{0}: G \rightarrow G$ defines an outer automorphism of $\pi_{1}(G, \tau(*)) \cong F_{N}$.

Consider $\Phi \in \operatorname{Out}\left(F_{N}\right)$. A topological representative of $\Phi$ is an homotopy equivalence $f_{0}: G \rightarrow G$, where $G$ is a marked graph, such that:

- the image of a vertex is a vertex,

- the image of an edge is a path,

- $f_{0}$ induces $\Phi$ on $F_{N} \cong \pi_{1}(G, \tau(*))$.

A topological representative $f_{0}: G \rightarrow G$ induces a map $f_{0 \#}$ on the finite paths of $G$ : if $w$ is a finite path, $f_{0 \#}(w)=\left[f_{0}(w)\right]$. If $f$ is some lift of $f_{0}$ to the universal cover $\tilde{G}$, and $w$ a finite path of $\tilde{G}, f_{\#}(w)$ is defined to be $[f(w)]$. Note that if $w$ is a finite path of $G, \tilde{w}$ a lift of $w$ in $\tilde{G}$, and $f$ a lift of $f_{0}$ to $\tilde{G}$, then $f_{0 \#}(w)$ is obtained by composing $f_{\#}(w)$ with the projection $p r: \tilde{G} \rightarrow G$.

2.2.2. Mating. In this paragraph, I recall elementary facts of covering theory applied to topological representatives; see for instance section 2.2 of [12] or section 2 of [4]. If $f_{0}: G \rightarrow G$ is some topological representative of $\Phi \in \operatorname{Out}\left(F_{N}\right)$, there exists a natural bijection between the sets of lifts $f$ of $f_{0}$ to the universal cover $\tilde{G}$ and the automorphisms $\varphi$ in the outer class $\Phi$ of $\varphi$. An automorphism $\varphi \in \Phi$ and a lift $f$ of $f_{0}$ are in correspondance if, and only if,

$$
\varphi(g) f=f g \quad \forall g \in F_{N},
$$

where the elements of $F_{N}$ are considered as deck transformations of $\tilde{G}$. We say that $\varphi$ and $f$ are mated.

Remark 2.1. Consider some mated lift $f$ and automorphism $\varphi$.

- Let $g \in F_{N}$. Then $g \in \operatorname{Fix} \varphi$ if and only if $g$ and $f$ commute.

- Using the natural identification of $\partial \tilde{G}$ and $\partial F_{N}$, one can see that $\partial f$ agrees with $\partial \varphi$.

2.2.3. Induced map on infinite paths. Let $f$ be some lift of $f_{0}$ and $\varphi \in \Phi$ be the automorphism mated with $f$. If $v$ is a point of $\tilde{G}$ and $R$ a ray starting at $v$ and representing an element $X \in \partial F_{N}$, then $f_{\#}(R)$ is defined to be the ray starting at $f(v)$ which represents $\partial \varphi(X)$. If $v_{0}$ is a point of $G$ and $R_{0}$ a ray starting at $v_{0}$ in $G$, $f_{0 \#}\left(R_{0}\right)$ is defined to be the composition of $f_{\#}(R)$ by the projection $p r: \tilde{G} \rightarrow G$, where $R$ is any lift of $R_{0}$ in $\tilde{G}$. If $l$ is a line of $\tilde{G}$ and $X_{1}, X_{2}$ the two points of $\partial F_{N}$ defined by this line, then $f_{\#}(l)$ is defined to be the line in $\tilde{G}$ defined by the two distinct points $\partial \varphi\left(X_{1}\right), \partial \varphi\left(X_{2}\right) \in \partial F_{N}$. If $l_{0}$ is a line in $G, f_{0 \#}\left(l_{0}\right)$ is defined to be the composition of $f_{\#}(l)$ by the projection $p r$, where $l$ is some lift of $l_{0}$ in $\tilde{G}$.

Alternatively, the image $f_{\#}(R)$ of a ray $R$ in $\tilde{G}$ can be viewed as the limit of the images by $f_{\#}$ of an increasing sequence of initial subpaths of $R$ (and similarly for lines).

\subsection{Train-tracks.}

2.3.1. Train-track maps. Consider $\Phi \in \operatorname{Out}\left(F_{N}\right)$ and a topological representative $f_{0}: G \rightarrow G$ of $\Phi$. A path $w$ in $\tilde{G}$ is legal if for some (and hence any) lift $f$ of $f_{0}$ and for all $k \geqslant 1$ :

$$
f^{k}(w)=f_{\#}^{k}(w) .
$$

Similarly, a path in $G$ is legal if any of its lifts at $\tilde{G}$ are legal.

The topological representative $f_{0}$ is a train-track map if $G$ has no vertex of valence 
1 or 2 , and any edge $e$ of $G$ is a legal path.

A Nielsen path of $G$ is a finite non-trivial path $\rho$ such that $f_{0 \#}(\rho)=\rho$.

The Theorem 1.7 of [4] states that every iwip outer automorphism $\Phi \in$ $\operatorname{Out}\left(F_{N}\right)$ can be represented by a train-track map $f_{0}: G \rightarrow G$. Moreover, there exists at most one Nielsen path $\rho$ in $G$.

2.3.2. Splittings. Let $f_{0}: G \rightarrow G$ be some topological representative of an outer automorphism $\Phi$, and $w$ a path in $G$. We say that $w=\ldots w_{l-1} w_{l} \ldots$, where the $w_{l}$ are non-trivial subpaths of $w$, is a splitting (for $f_{0}$ ), and then we write $w=\ldots \cdot w_{l-1} \cdot w_{l} \cdot \ldots$, if for all $k \geqslant 1, f_{0 \#}^{k}(w)=\ldots f_{0 \#}^{k}\left(w_{l-1}\right) f_{0 \#}^{k}\left(w_{l}\right) \ldots$ (a concatenation without tigthtening). In this case, the $w_{l}$ are called the bricks of $w$.

Let $f$ be a lift of $f_{0}$ to the universal cover $\tilde{G}$, and $w$ a path in $\tilde{G}$. Similarly, we say that $w=\ldots w_{l-1} w_{l} \ldots$, where the $w_{l}$ are non-trivial subpaths of $w$, is a splitting (for $f$ ), and then we write $w=\ldots \cdot w_{l-1} \cdot w_{l} \cdot \ldots$, if for all $k \geqslant 1$, $f_{\#}^{k}(w)=\ldots f_{\#}^{k}\left(w_{l-1}\right) f_{\#}^{k}\left(w_{l}\right) \ldots$; the $w_{l}$ are still called bricks of $w$.

Definition 2.1. Let $\Phi \in \operatorname{Out}\left(F_{N}\right)$ be an iwip outer automorphism. A train-track map $f_{0}: G \rightarrow G$ representing $\Phi$ is said to be appropriate if it satisfies the following property. For any lift $f: \tilde{G} \rightarrow \tilde{G}$ of $f_{0}$ and for any finite path $w$ in $\tilde{G}$, there exists some positive integer $K$ such that for all $k \geqslant K, f_{\#}^{k}(w)$ has a splitting where the bricks are either

- edges, or

- lifts of the Nielsen path $\rho$ of $f_{0}$ (if exists).

The following lemma, which will be useful for us, can be alternatively found in [3] (lemma 4.2.6) or in [17] (lemma 3.2) in a more general setting; see also [6].

Lemma 2.1. Let $\Phi \in \operatorname{Out}\left(F_{N}\right)$ be an iwip outer automorphism. Then there exists some positive power of $\Phi$ which can be represented by an appropriate train-track map.

Remark 2.2. Consider a path $w$ in $\tilde{G}$ which has a splitting whose bricks are either edges or lifts of the Nielsen path, and $x, y$ two points in $w$ with $x \leqslant y$ (where $\leqslant$ is the order induced by the orientation of $w)$. We remark that if $f^{k}(x), f^{k}(y) \in f_{\#}^{k}(w)$ for some $k \geqslant 1$, then $f^{k}(x) \leqslant f^{k}(y)$ in $f_{\#}^{k}(w)$. Indeed, this is clear if $w$ is an edge and classical if $w$ is a lift of the Nielsen path (see lemma 3.4 of [4]); then the result follows by the definition of a splitting.

\section{The ATtRACTIVE LAMinATION OF AN IWIP AUTOMORPHISM}

In this section, I just give some material about laminations in free groups which will be used later in this article. For a detailed and more complete exposition, the reader is refered to [9].

3.1. Laminations. Let us consider the set $\partial^{2} F_{N}=\partial F_{N} \times \partial F_{N} \backslash \Delta$ (where $\Delta$ is the diagonal in $\left.\partial F_{N} \times \partial F_{N}\right)$ of pairs of distinct points in $\partial F_{N}$. Note that the topology of $\partial F_{N}$ induces a topology on $\partial^{2} F_{N}$. Moreover, the action of $F_{N}$ on $\partial F_{N}$ induces a diagonal action of $F_{N}$ on $\partial^{2} F_{N}$. The set $\partial^{2} F_{N}$ admits a distinguished involution $(X, Y) \mapsto(Y, X)$, called the flip involution: it is an $F_{N}$-equivariant homeomorphism.

An algebraic lamination $L$ of $F_{N}$ is a subset of $\partial^{2} F_{N}$, which is closed, $F_{N^{-}}$ invariant and flip-invariant. 
If we are given some marked graph $G$ and a base point of its universal cover $\tilde{G}$, recall that a point in $\partial^{2} F_{N}$ defines a unique line in $\tilde{G}$. Thus an algebraic lamination $L$ defines a set of lines in $\tilde{G}$, denoted by $L(\tilde{G})$. The symbolic lamination in $G$-coordinates associated to $L$, denoted by $L(G)$, is the set of lines in $G$ which can be lifted to lines of $L(\tilde{G})$. The lines of $L(G)$ are called leaves.

To such a symbolic lamination $L(G)$, one can associate the set $\mathcal{L}(L(G))$, called the laminary language of $L$ in $G$-coordinates, which consists of all the finite edge paths in $G$ which occur in some leaf of $L(G)$.

3.2. Action of Out $\left(F_{N}\right)$ on the laminations. The group $\operatorname{Out}\left(F_{N}\right)$ acts naturally on the set of algebraic laminations of $F_{N}$, in the following way. Consider $\varphi \in$ $\operatorname{Aut}\left(F_{N}\right)$ and $(X, Y) \in \partial^{2} F_{N}$, and define $\partial^{2} \varphi((X, Y))$ to be $(\partial \varphi(X), \partial \varphi(Y))$; then $\partial^{2} \varphi$ is a homeomorphism of $\partial^{2} F_{N}$. Notice that, if $L$ is an algebraic lamination, then $\partial^{2} \varphi(L)$ is also an algebraic lamination. Moreover, since $L$ is $F_{N}$-invariant, $\partial^{2} \varphi(L)$ only depends of the outer class $\Phi$ of $\varphi$, and we shall denote it $\Phi(L)$.If $\Phi(L)=L$, one traditionally says that $\Phi$ stabilizes the lamination $L$.

Now consider a topological representative $f_{0}: G \rightarrow G$ of an outer automorphism $\Phi \in \operatorname{Out}\left(F_{N}\right)$. Denote by $C$ the cancellation bound of $f_{0}$ : it is the smallest constant $C>0$ such that for any lift $f$ of $f_{0}$ to $\tilde{G}$, and for any path $w$ of $\tilde{G}, f(w)$ is contained in a $C$-neighbourhood of $f_{\#}(w)$. The existence of a cancellation bound has been first established in [8] in the special case of a rose, and then generalised for general marked graphs (see [3] for instance).

If $w$ is a finite edge path in $G$, we denote $f_{0 \#, C}(w)$ the longest sub-edge-path of the path obtained by removing both extremities of length $C$ of $f_{0 \#}(w)$. If $L$ is an algebraic lamination, we say that $f_{0}$ stabilizes the laminary language $\mathcal{L}(L(G))$ if for all $w \in \mathcal{L}(L(G)), f_{0 \#, C}(w) \in \mathcal{L}(L(G))$.

3.3. The attractive lamination of an iwip outer automorphism. Consider a graph $G$ endowed with the simplicial metric (i.e. every edge is isometric to the segment $[0,1]$ ). A line $l$ in $G$ is said quasiperiodic if for all $L>0$ there exists $L^{\prime}>L$ such that every subpath of length $L$ of $l$ occurs as a subpath of every subpath of length $L^{\prime}$ of $l$. Equivalentely, a line $l$ of $G$ is quasiperiodic if for all subpath $m$ of $l$, there exists $K>0$ such that $m$ occurs infinitely often in (both ends of) $l$, and the distance between two successive occurences of $m$ is bounded by $K$ : we say that $l$ has the bounded gap property.

Consider an iwip outer automorphism $\Phi$, and some train-track representative $f_{0}: G \rightarrow G$. Define the set $\mathcal{L}_{f_{0}}^{+}$of finite edge paths in $G$ by the following condition: an edge path $w$ of $G$ is in $\mathcal{L}_{f_{0}}^{+}$if, and only if, there exists an edge $e$ of $G$ and an integer $k \geqslant 1$ such that $w$ is a subpath of $f_{0}^{k}(e)$.

It is proved in [2] that:

\section{Proposition 3.1.}

i) For any edge e of $G$ and for all $w$ in $\mathcal{L}_{f_{0}}^{+}$, there exists an integer $k \geqslant 1$ such that $w$ is a subpath of $f_{0}^{k}(e)$.

ii) There exists an algebraic lamination $L_{\Phi}^{+}$, called the attractive (algebraic) lamination of $\Phi$, whose laminary language in $G$-coordinates is $\mathcal{L}_{f_{0}}^{+}$.

iii) This algebraic lamination $L_{\Phi}^{+}$does not depend on the choice of the train-track map representing $\Phi$.

iv) Every leaf of the attractive (symbolic) lamination $L_{\Phi}^{+}(G)$ is quasiperiodic. 
Moreover, the following important result is proved in [2]:

Theorem 3.2 (Bestvina-Feighn-Handel). If $\Phi \in \operatorname{Out}\left(F_{N}\right)$ is an iwip outer-automorphism, then the stabilizer $\operatorname{Stab}\left(L_{\Phi}^{+}\right)$of $L_{\Phi}^{+}$in Out $\left(F_{N}\right)$ is virtually infinite cyclic.

The following lemma gives a concrete criterion to prove that an outer automorphism stabilizes the attractive lamination of another outer automorphism:

Lemma 3.3. Let $\Phi \in \operatorname{Out}\left(F_{N}\right)$ be an iwip outer-automorphism and $L_{\Phi}^{+}$be its attractive lamination. Then $\Psi \in \operatorname{Out}\left(F_{N}\right)$ stabilizes $L_{\Phi}^{+}$if and only if there exists some topological representative $g_{0}: G \rightarrow G$ of $\Psi$ which stabilizes the laminary language $\mathcal{L}\left(L_{\Phi}^{+}, G\right)$ of $\Phi$.

One can find the substance of the proof of this lemma in section 3 of [2]. But it is a general fact that a general fact that an outer automorphism stabilizes a lamination if, and only if, it stabilizes its laminary language (see [9]).

\section{Attractive FIXed POINT OF AN IWIP AUtOMORPhism}

\subsection{Structure of an attractive fixed point of an iwip automorphism.}

Proposition 4.1. Let $\Phi \in \operatorname{Out}\left(F_{N}\right)$ be an iwip outer automorphism which can be represented by an appropriate train-track map $f_{0}: G \rightarrow G$. Let $\varphi \in \Phi$, and let $f$ be the lift of $f_{0}$ mated with $\varphi$, and $X \in \partial F_{N}$ be an attractive fixed point of $\varphi$. Then there exists a vertex $v$ in $\tilde{G}$ such that:

i) $\left[v, f^{2}(v)\right]=[v, f(v)] \cdot\left[f(v), f^{2}(v)\right]$;

ii) the ray $R_{v}=[v, f(v)] \cdot\left[f(v), f^{2}(v)\right] \cdot \ldots \cdot\left[f^{k}(v), f^{k+1}(v)\right] \cdot \ldots$ represents the point $X$;

iii) the segment $[v, f(v)]$ has a splitting whose bricks are either an edge or a lift of the Nielsen path $\rho$ of $G$ (if it exists); moreover, the first brick of this splitting is an edge.

Proof. First step: We will show that there exists a point $v$ in $\tilde{G}$ which satisfies properties i) and ii) of Proposition 4.1. Since $X$ is an attractive fixed point of $\partial \varphi$, there exists a vertex $v_{0}$ of $\tilde{G}$ (sufficiently close to $X$ ) such that $\lim _{k \rightarrow+\infty} f^{k}\left(v_{0}\right)=$ $X$. We denote by $R_{v_{0}}$ the ray starting at $v_{0}$ which represents $X$. Let's define $v_{k+1}$ by induction as the projection of $f\left(v_{k}\right)$ on $R_{v_{0}}$. Note that $\left[v_{k+1}, v_{k+2}\right] \subseteq$ $\left[f\left(v_{k}\right), f\left(v_{k+1}\right)\right]=f_{\#}\left(\left[v_{k}, v_{k+1}\right]\right) \subseteq f\left(\left[v_{k}, v_{k+1}\right]\right)$.

Let us denote $V_{k}=\left\{v \in \tilde{G}: f^{i}(v) \in\left[v_{i}, v_{i+1}\right], \forall 0 \leqslant i \leqslant k\right\}$. It is clear that $f^{k}\left(V_{k}\right) \subseteq\left[v_{k}, v_{k+1}\right]$; we show by induction that, in fact, this inclusion is an equality. Indeed, $V_{0}=\left[v_{0}, v_{1}\right]$. Suppose that $f^{k}\left(V_{k}\right)=\left[v_{k}, v_{k+1}\right]$. Since $\left[v_{k+1}, v_{k+2}\right] \subseteq f\left(\left[v_{k}, v_{k+1}\right]\right)$, we obtain that $\left[v_{k+1}, v_{k+2}\right] \subseteq f^{k+1}\left(V_{k}\right)$. We deduce that $\left[v_{k+1}, v_{k+2}\right] \subseteq f^{k+1}\left(V_{k+1}\right)$, since $x \in V_{k+1}$ if and only if $x \in V_{k}$ and $f^{k+1}(x) \in\left[v_{k+1}, v_{k+2}\right]$. In particular, all of the $V_{k}$ are non-empty. Since for all $k$, the set $V_{k}$ is obviously closed, the $V_{k}$ form a sequence of non-empty compact subsets of $\left[v_{0}, v_{1}\right]$, which implies that $\cap_{k \in \mathbb{N}} V_{k} \neq \emptyset$. Choose some point $v \in \cap_{k \in \mathbb{N}} V_{k}$. Then $v$ satisfies properties i) and ii) (since for all $k, f^{k}(v) \in\left[v_{k}, v_{k+1}\right]$ ).

Second step: We would like $v$ to be a vertex. If this is not the case, we argue as follows. Thanks to Lemma 2.1, up to replacing $v_{0}$ by some $f^{k}\left(v_{0}\right)$, we can suppose that the path $u=\left[v_{0}, f^{2}\left(v_{0}\right)\right]$ has a splitting whose bricks are either edges or lifts of the Nielsen path $\rho$ of $f_{0}$ (if it exists). Then consider the initial vertex $v^{\prime}$ of the brick of $u$ that contains $f(v)$. Since $v^{\prime}$ is a splitting point of $u$, one has 
$f^{k}\left(v^{\prime}\right) \in f_{\#}^{k}(u)=\left[f^{k}\left(v_{0}\right), f^{k+2}\left(v_{0}\right)\right]$ for all $k \geqslant 1$, and hence, since $v^{\prime} \in[v, f(v)]$, one has $f^{k}\left(v^{\prime}\right) \in\left[f^{k}(v), f^{k+1}(v)\right]$ (see Remark 2.2). This implies that $f^{k}\left(v^{\prime}\right) \in R_{v_{0}}$ for all $k \geqslant 1$. Thus we have found a vertex $v^{\prime}$ satisfies properties i) and ii).

Moreover, the fact that $X$ is an attractive fixed point of $\partial \tilde{f}$ implies that $\lim _{k \rightarrow+\infty} d\left(f^{k}\left(v^{\prime}\right), f^{k+1}\left(v^{\prime}\right)\right)=+\infty$ (see [12] or [16]). We deduce that there exists a brick $b$ of $\left[v^{\prime}, f\left(v^{\prime}\right)\right]$ such that $\lim _{k \rightarrow+\infty}\left|f_{\#}^{k}(b)\right|=+\infty$ : so $b$ must be an edge. Replacing $v^{\prime}$ by the initial point of $b$, we have proved property iii), while i), ii) are still satisfied.

In the splitting of $[v, f(v)]$, we group together successive bricks which are lifts of $\rho$. We obtain a splitting $[v, f(v)]=b_{0} \cdot b_{1} \cdot \ldots \cdot b_{q}$ whose bricks are either:

- a single edge (in which case, we say that $b_{i}$ is regular), or

- a lift of some power $\rho^{r_{i}}\left(r_{i} \in \mathbb{Z} \backslash\{0\}\right)$ of the Nielsen path (we then say that $b_{i}$ is singular).

Note that $b_{0}$ is regular, and that between 2 singular bricks there is at least one regular brick. We call this splitting the adapted splitting of $[v, f(v)]$. By setting $b_{i, k}=f_{\#}^{k}\left(b_{i}\right)(0 \leqslant i \leqslant q, k \in \mathbb{N})$, we obtain a splitting $\left[f^{k}(v), f^{k+1}(v)\right]=b_{0, k} \cdot b_{1, k}$. $\ldots \cdot b_{q, k}$.

Definition 4.1. With the previous notations, we define the adapted splitting of $R_{v}$ as the splitting by the bricks $b_{i, k}$. When $b_{i}$ is regular (resp. singular), we say that $b_{i, k}$ is regular (resp. singular) as well.

Remark 4.1. Between two singular bricks of the adapted splitting of $R_{v}$, there is at least a regular brick (since this is the case for each $\left[f^{k}(v), f^{k+1}(v)\right]$, and since the first brick of any $\left[f^{k}(v), f^{k+1}(v)\right]$ is regular). Note that the singular bricks of $R_{v}$ have length bounded above by the length of the longest singular brick of $[v, f(v)]$.

\subsection{The stabilizer of an attractive fixed point of an iwip automorphism.}

Theorem 4.2. If $X \in \partial F_{N}$ is an attractive fixed point of an iwip automorphism $\varphi \in \operatorname{Aut}\left(F_{N}\right)$, and if $\psi \in A u t\left(F_{N}\right)$ fixes $X$, then the outer automorphism $\Psi$ defined by $\psi$ stabilizes the attractive lamination $L_{\Phi}^{+}$of the outer automorphism $\Phi$ defined by $\varphi$.

Proof. We remark that an attractive fixed point $X$ of $\varphi$ is also an attractive fixed point of any positive power of $\varphi$, and that the attractive lamination $L_{\Phi}^{+}$of $\Phi$ is equal to the the attractive lamination of any positive power of $\Phi$. Thus, up to replacing $\varphi$ by a positive power, we can suppose that $\Phi$ can be represented by an appropriate train-track map $f_{0}: G \rightarrow G$ (see Lemma 2.1).

We consider a topological representative $g_{0}: G \rightarrow G$ of $\Psi$, on the same graph $G$. We denote by $C \geqslant 0$ the cancellation bound of $g_{0}$. We denote by $f$ (or $g$ ) the lift of $f_{0}$ (or $g_{0}$ ) to $\tilde{G}$ mated with $\varphi$ (or $\psi$ ). We choose a ray $R_{v}$ in $\tilde{G}$ representing $X$ as in proposition 4.1, endowed with its adapted splitting as defined above. We denote by $l_{0}$ the maximal length of a singular brick of $R_{v}$ (see Remark 4.1).

Consider an arbitrary edge path $u$ in the laminary language $\mathcal{L}\left(L_{\Phi}^{+}(G)\right)$ of the symbolic attractive lamination of $\Phi$. We are going to show that there exists an occurence of $g_{0 \#, C}(u)$ in $R_{v}$ which is completely contained in a regular brick. Then $g_{0 \#, C}(u)$ is also contained in $\mathcal{L}\left(L_{\Phi}^{+}(G)\right)$, and according to Lemma 3.3 , the proposition is proved. 
Since every leaf of $L_{\Phi}^{+}(G)$ is quasiperiodic (see Proposition 3.1), we can find an edge path $U \in \mathcal{L}\left(L_{\Phi}^{+}(G)\right)$ of type $U=u u_{0} u$, where $u_{0}$ is an edge path of length greater than every constant previously fixed. In particular, we can choose $u_{0}$ so that:

$$
\left|g_{0 \#}\left(u_{0}\right)\right| \geqslant l_{0}+2 C \text {. }
$$

Indeed, since $g$ is a quasi-isometry, there exist positive constants $\mu$ and $\nu$ such as $\mu^{-1}|m|-\nu \leqslant\left|g_{0 \#}(m)\right| \leqslant \mu|m|+\nu$ for any path $m$. Hence it suffices to choose a path $u_{0}$ of length greater than $\mu\left(l_{0}+2 C+\nu\right)$.

By definition of $\mathcal{L}\left(L_{\Phi}^{+}\right)$, there exists an integer $K \in \mathbb{N}$ such that in all regular bricks $b_{i, k}$ with $k \geqslant K$, there is an occurence of $U$. In particular, there are infinitely many occurences of $U$ in $R_{v}$, and hence infinitely many occurences of $g_{0 \#, C}(U)$ in $g_{0 \#}\left(R_{v}\right)$. Since $\psi(X)=X, g_{0 \#}\left(R_{v}\right) \cap R_{v}$ is a subray of $R_{v}$, and thus there are infinitely many occurences of $g_{0 \#, C}(U)$ in $R_{v}$ : we denote by $\left(w_{j}\right)$ a sequence of (distinct) occurences $g_{0 \#, C}(U)$ in $R_{v}$.

Remark 4.2. If $m$ is a path, there is only a finite number of occurences of $m$ in $R_{v}$ which fully contains a regular brick (since there exists a subray $R$ of $R_{v}$ such that every regular brick contained in $R$ is of length greater than $|m|)$.

If there exists $j \in \mathbb{N}$ such that $w_{j}$ is fully contained in a regular brick, then there exists an occurence of $g_{0 \#, C}(u)$ in this brick (since $g_{0 \#, C}(u)$ appears as a subpath of $\left.g_{0 \#, C}(U)\right)$, and our claim is proved. Otherwise, according to Remark 4.2 , all but finitely many of the $w_{j}$ meet at most two regular bricks and a singular brick of $R_{v}$. More precisely, up to replacing $\left(w_{j}\right)$ by an infinite subsequence, we can suppose that one of the following properties is satisfied:

i) all of the $w_{j}$ meet two regular bricks and the singular brick joining them,

ii) all of the $w_{j}$ meet two consecutive bricks: a regular one and a singular one,

iii) all of the $w_{j}$ meet two consecutive regular bricks.

In any of the three cases, we can suppose that the points of the adapted splitting of $R_{v}$ appear at the same place in all of the $w_{j}$ (since $g_{0 \#, C}(U)$ is a finite path).

In case i) one has $g_{0 \#, C}(U)=u_{1} \cdot b \cdot u_{2}$ where the $u_{i}$ are contained in regular bricks, and $b$ is a singular brick. There exists $i \in\{1,2\}$ such that $\left|u_{i}\right| \geqslant$ $\left(\left|g_{0 \#, C}(U)\right|-l_{0}\right) / 2$. Moreover, $\left|g_{0 \#, C}(U)\right| \geqslant 2\left|g_{0 \#, C}(u)\right|+\left|g_{0 \#, C}\left(u_{0}\right)\right|$ since $U=$ $u u_{0} u$. Using (1) we obtain $\left|u_{i}\right| \geqslant\left|g_{0 \#, C}(u)\right|$. Thus $g_{0 \#, C}(u)$ occurs as a subpath of a regular brick.

In case ii) suppose, for instance, that the regular brick precedes the singular one: then $g_{0 \#, C}(U)=u_{1} \cdot b^{\prime}$ with $u_{1}$ contained in a regular brick and $b^{\prime}$ in a singular brick for all occurences of $g_{0 \#, C}(U)$. Then $\left|u_{1}\right| \geqslant\left|g_{0 \#, C}(U)\right|-l_{0} \geqslant\left|g_{0 \#, C}(u)\right|$, and thus $g_{0 \#, C}(u)$ occurs as a subpath of a regular brick.

In case iii) one has $g_{0 \#, C}(U)=u_{1} \cdot u_{2}$ where the $u_{i}$ are contained in regular bricks, and the splitting point corresponds to a splitting point of the adapted splitting of $R_{v}$. As in case i), we obtain that there exists $i \in\{1,2\}$ such that $\left|u_{i}\right| \geqslant g_{0 \#, C}(U) / 2 \geqslant\left(2\left|g_{0 \#, C}(u)\right|+\left|g_{0 \#, C}\left(u_{0}\right)\right|\right) / 2 \geqslant\left|g_{0 \#, C}(u)\right|$. We conclude once again that $g_{0 \#, C}(u)$ occurs as a subpath of a regular brick.

The following proposition shows that a periodic automorphism can not fix an attractive fixed point of an iwip automorphism. 
Proposition 4.3. Let $X \in \partial F_{N}$ be an attractive fixed point of an iwip automorphism $\varphi \in \operatorname{Aut}\left(F_{N}\right)$. If $\psi \in \operatorname{Aut}\left(F_{N}\right)$ is a periodic automorphism which fixes $X$, then $\psi$ is the identity.

Proof. Indeed, since $\psi$ is periodic, the fixed points of $\partial \psi$ are singular (cf Lemma 1.3 ), and according to [10], Fix $\psi$ is a free factor of $F_{N}$. In particular, if $\psi$ is not the identity, then Fix $\psi$ has infinite index in $F_{N}$, and thus Proposition 2.4 of [2] implies that $\operatorname{Fix} \psi$ does not carry the attractive lamination of $\Phi$. This means (see section 2 of [2] for more details) that if $f_{0}: G \rightarrow G$ is a topological representative of $\Phi$, and $\tau: G^{\prime} \rightarrow G$ a graph immersion (that is a locally injective map) such that $\pi_{1}(\operatorname{Im}(\tau))=$ Fix $\psi$, then there exists a leaf $l$ of $L_{\Phi}^{+}(G)$ which does not lift to $G^{\prime}$. In particular, there exists a (sufficiently long) finite subpath $w$ of $l$ which can not be lifted to $G^{\prime}$.

Let $R_{v}$ be a ray in $G$ representing $X$, as in proposition 4.1. By the definition of $L_{\Phi}^{+}, w$ appears in all sufficiently long regular bricks of $R_{v}$. In particular, $w$ occurs infinitely many times in $R_{v}$, and thus also in any ray $R$ of $G$ representing $X$. Hence no ray $R$ representing $X$ can be lifted to $G^{\prime}$. Thus $X$ cannot be fixed by $\partial \varphi$.

The following corollary of Theorems 3.2 and 4.2, and Proposition 4.3, proves Theorem I stated in the Introduction.

Corollary 4.4. Let $\Phi \in \operatorname{Out}\left(F_{N}\right)$ an iwip outer automorphism. If $X \in \partial F_{N}$ is an attractive fixed point of an iwip automorphism $\varphi \in \Phi$, then Stab $(X)$ injects into $\operatorname{Stab}\left(L_{\Phi}^{+}\right)$via the quotient map Aut $\left(F_{N}\right) \rightarrow \operatorname{Out}\left(F_{N}\right)$. Moreover, Stab $(X)$ is infinite cyclic.

Proof. As an immediate consequence of Theorem 4.2 and Proposition 1.2, we obtain that $\operatorname{Stab}(X)$ injects into $\operatorname{Stab}\left(\Lambda_{\Phi}^{+}\right)$. Using Theorem 3.2, we obtain that $\operatorname{Stab}(X)$ is virtually infinite cyclic. Hence proposition 4.3 allows to conclude that $\operatorname{Stab}(X) \cong \mathbb{Z}$.

\section{Subgroups of $\operatorname{Aut}\left(F_{2}\right)$ fixing a point of $\partial F_{2}$}

\subsection{Basic facts about $\operatorname{Out}\left(F_{2}\right)$ and $\operatorname{Aut}\left(F_{2}\right)$.}

5.1.1. Nielsen's identifications. Every outer automorphism $\Phi \in \operatorname{Out}\left(F_{N}\right)$ induces, by abelianisation, an automorphism of $\mathbb{Z}^{N}$. This defines an homomorphism of groups:

$$
\begin{aligned}
A b: \operatorname{Out}\left(F_{N}\right) & \rightarrow G l_{N}(\mathbb{Z}) \\
\Phi & \mapsto M .
\end{aligned}
$$

We denote by $\operatorname{Out}^{+}\left(F_{N}\right)$ the preimage of $S l_{N}(\mathbb{Z})$.

Now consider an orientable surface $\Sigma$, possibly with non empty boundary. We denote by Homeo $(\Sigma)$ the group of homeomorphisms of $\Sigma$, by $\mathrm{Homeo}^{+}(\Sigma)$ the subgroup of the ones preserving the orientation and by $\mathrm{Homeo}_{0}(\Sigma)$ the subgroup of those homotopic to the identity. The mapping class group of $\Sigma$ is defined by $\operatorname{MCG}(\Sigma)=\operatorname{Homeo}^{+}(\Sigma) / \operatorname{Homeo}_{0}(\Sigma)$; we also define $\mathrm{MCG}^{ \pm}(\Sigma)=\mathrm{Homeo}_{(\Sigma)} / \mathrm{Homeo}_{0}(\Sigma)$.

If $\Sigma$ has non-empty boundary, then its fundamental group $\pi_{1}(\Sigma)$ is a free group $F_{N}$. Any element $f \in \operatorname{MCG}(\Sigma)$ induces an outer automorphism of $\pi_{1}(\Sigma)=$ $F_{N}$. This defines an injective group homomorphism

$$
\begin{array}{ccc}
J: \operatorname{MCG}^{ \pm}(\Sigma) & \rightarrow & \operatorname{Out}\left(F_{N}\right) \\
f & \mapsto & \Phi
\end{array} .
$$




\begin{tabular}{|c|c|c|}
\hline$f \in \operatorname{MCG}\left(F_{2}\right)$ & $\Phi \in \mathrm{Out}^{+}\left(F_{2}\right)$ & $M \in S l_{2}(\mathbb{Z})$ \\
\hline \hline pseudo-Anosov & iwip & $|\operatorname{tr}(M)|>2$ \\
\hline$f$ or $f^{2}$ Dehn-twist & linear growth & $M \neq \pm I_{2}$ and $|\operatorname{tr}(M)|=2$ \\
\hline finite order & finite order & $M= \pm I_{2}$ or $|\operatorname{tr}(M)|<2$ \\
\hline
\end{tabular}

TABLE 1. Correspondence between $\mathrm{MCG}(T), \mathrm{Out}^{+}\left(F_{2}\right), \mathrm{Sl}_{2}(\mathbb{Z})$

Now consider the surface $T$ obtained by removing an open disk from a torus. Then $\pi_{1}(T)=F_{2}$. A celebrated result of J. Nielsen ( $\left.\operatorname{cf}[19]\right)$ says that the morphisms

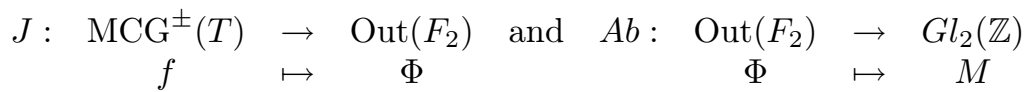

are in fact isomorphisms. Of course, we obtain isomorphisms by restriction to $\operatorname{MCG}(T), \mathrm{Out}^{+}\left(F_{2}\right)$ and $\mathrm{Sl}_{2}(\mathbb{Z})$.

Thus, to study $\mathrm{Out}^{+}\left(F_{2}\right)$, we can use alternatively the celebrated NielsenThurston classification of homeomorphisms of surfaces (see [11] or [7]), or classical results on $S L_{2}(\mathbb{Z})$ : In table 1 , the correspondence between these different view points is given (cf the introduction of [7] or the expose 1 of [11]).

When $f \in \mathrm{MCG}^{ \pm}(T)$ is a Dehn twist (resp. a pseudo-Anosov homeomorphism, ...), we also say that the corresponding $\Phi \in \operatorname{Out}\left(F_{2}\right)$ and $M \in G l_{2}(\mathbb{Z})$ are Dehn twists (...).

5.1.2. Periodic outer automorphisms. Consider a finite order element $\Phi \in \operatorname{Out}\left(F_{2}\right)$, and let $\varphi \in \Phi$. Then there exists $p \geqslant 1$ such that $\Phi^{p}=I d$, and thus there exists $u \in F_{2}$ such that $\varphi^{p}=i_{u}$.

If $u \neq 1$, then $\operatorname{Fix} \varphi^{p}=<\sqrt{u}>$, and the fixed points of $\partial \varphi^{p}$ are $u^{-\infty}$ and $u^{\infty}$. Thus $\partial \varphi$ has either no fixed point, or two fixed points: $u^{ \pm \infty}$.

Otherwise $\varphi^{p}=i d$. This implies that:

- Fix $\varphi$ is a free factor of $F_{2}$ (this is a general fact, proved by J. Dyer and G. Scott, which states that the fixed subgroup of a periodic atutomorphism of $F_{N}$ is a free factor; cf [10]);

- the fixed points of $\partial \varphi$ must be singular.

If $\operatorname{Fix} \varphi$ is trivial, then $\partial \varphi$ has no fixed points. If $\operatorname{Fix} \varphi$ has rank one, let us denote by $u$ some generator of $\operatorname{Fix} \varphi$. Then $\operatorname{Fix} \partial \varphi=\left\{u^{ \pm \infty}\right\}$. The case where $\operatorname{Fix} \varphi$ has rank two corresponds to $\varphi=i d$, since $\operatorname{Fix} \varphi$ is a free factor of $F_{2}$.

Thus we have proved:

Lemma 5.1. Consider $\varphi \in \operatorname{Aut}\left(F_{2}\right), \varphi \neq i d, X \in \partial F_{2}$ a fixed point of $\partial \varphi$, and $\Phi \in \operatorname{Out}\left(F_{2}\right)$ the outer class of $\varphi$. If $\Phi$ is periodic, then $X$ is rational.

5.1.3. Dehn twists. A Dehn twist in $G l_{2}(\mathbb{Z})$ can be represented by a matrix $D=$ $\left(\begin{array}{ll}1 & p \\ 0 & 1\end{array}\right)(p \in \mathbb{Z}, p \neq 0)$. Remark that $D=D_{0}^{p}$, with $D_{0}=\left(\begin{array}{ll}1 & 1 \\ 0 & 1\end{array}\right)$. The centralizer of $M \in G L_{2}(\mathbb{Z})$, denoted by $C e n_{G l_{2}(\mathbb{Z})}(D)$, is the subgroup of $G l_{2}(\mathbb{Z})$ of all elements commuting with $M$. The following lemma, which sums up usual properties of roots and centralizer of a Dehn twist, results from simple calculations in $G l_{2}(\mathbb{Z})$ (alternatively, it can be proved using surface homeomorphisms theory see for instance [14]). 
Lemma 5.2. Let $D_{0}=\left(\begin{array}{ll}1 & 1 \\ 0 & 1\end{array}\right)$, and $D=D_{0}^{p}(p \in \mathbb{Z}, p \neq 0)$.

i) The matrix $D$ has a square root in $G l_{2}(\mathbb{Z})$ if, and only if $p$ is even. In this case, $D$ has exactly two square roots: $\pm D_{0}^{p / 2}$, and both belong to $S l_{2}(\mathbb{Z})$.

ii) The matrix $-D_{0}^{p}$ has no square root.

iii) $\operatorname{Cen}_{G L_{2}(\mathbb{Z})}( \pm D)=<D_{0},-I_{2}>\cong \mathbb{Z} \oplus \mathbb{Z} / 2 \mathbb{Z}$.

iv) One assumes that $|p|>4$. If $D^{\prime}$ is a Dehn twist (or a root of a Dehn twist) which does not commute with $D$, then $D D^{\prime}$ is pseudo-Anosov.

Remark 5.1. Point iv) of lemma 5.2 can be false if we do not assume $|p|>4$. For instance, $D=D_{0}^{4}$ and $D^{\prime}=\left(\begin{array}{cc}1 & -4 \\ 1 & -3\end{array}\right)$ do not commute and yet, $D D^{\prime}=$ $\left(\begin{array}{cc}5 & -16 \\ 1 & -3\end{array}\right)$ is a Dehn twist.

5.2. The stabilizer of a point of $\partial F_{2}$. The aim of this section is to determine which are the maximal subgroups of $\operatorname{Aut}\left(F_{2}\right)$ that fix a point $X \in \partial F_{2}$.

If $X \in \partial F_{2}$ is not a rational point, and if there exists a non-trivial automorphism $\varphi \in \operatorname{Aut}\left(F_{2}\right)$ fixing $X$, then:

- either the outer automorphism $\Phi$ is iwip,

- or $\Phi^{2}$ is a Dehn twist.

Otherwise, $\Phi$ would be periodic, and so, according to Lemma 5.1, $X$ would be rational.

Corollary 4.4 implies that, if $\varphi$ and $\psi$ both fix $X$, then either they are both iwip, or both $\Phi^{2}$ and $\Psi^{2}$ are Dehn twists. In the first case, Corollary 4.4 says that the stabilizer of $X$ is infinite cyclic. In the second case, $\Phi^{6}$ and $\Psi^{2}$ must commute, since otherwise point iv) of Lemma 5.2 implies that $\Phi^{6} \circ \Psi^{2}$ is an iwip automorphism fixing $X$, in contradiction to Corollary 4.4. Thus $\Psi \in C e n_{\text {Out }\left(F_{2}\right)}(\Phi) \cong \mathbb{Z} \oplus \mathbb{Z} / 2 \mathbb{Z}$ (see point iii) of Lemma 5.2). According to Proposition 1.2, $\operatorname{Stab}(X)$ injects into $\mathbb{Z} \oplus \mathbb{Z} / 2 \mathbb{Z}$. Moreover, according to Lemma 5.1 , since $X$ is not rational, if $\varphi \in$ $\operatorname{Stab}(X)$, then $\Phi$ is not periodic. As a consequence $\operatorname{Stab}(X)$ is infinite cyclic. Thus we have shown the:

Theorem 5.3. Let $X \in \partial F_{2}$ a non-rational point. Then the stabilizer of $X$ in $\operatorname{Aut}\left(F_{2}\right)$ is either trivial, or infinite cyclic.

If $X \in \partial F_{2}$ is a rational point, then $\operatorname{Stab}(X)=\operatorname{Stab}(\sqrt{X})$ (see Proposition 1.1). In [5], we can find a complete description of the stabilizers of the elements of $F_{2}$. Up to isomorphism, we obtain the groups $\mathbb{Z}, \mathbb{Z}^{2},\left\langle x, y\left|x^{2}=y^{2}>,<x, y\right| x^{2}=\right.$ $1,\left[x, y^{2}\right]=1>,\langle x, y| x y x=y x y>$.

Thus we obtain the following corollary, which ends the proof of Theorem II stated in the Introduction:

Corollary 5.4. Up to isomorphism, the maximal non-trivial subgroups of Aut $\left(F_{2}\right)$ which fix an element in the boundary of $F_{2}$ are the same as the ones fixing an element of $F_{2}$. They are given by

$$
\mathbb{Z}, \mathbb{Z}^{2},<x, y\left|x^{2}=y^{2}>,<x, y\right| x^{2}=1,\left[x, y^{2}\right]=1>,<x, y \mid x y x=y x y>.
$$




\section{REFERENCES}

1. P. Arnoux, V. Berthé, A. Hilion, and A. Siegel, Fractal representation of the attractive lamination of an automorphism of the free group, preprint available on http://junon.u3mrs.fr/hilion/, 2005.

2. M. Bestvina, M. Feighn, and M. Handel, Laminations, trees, and irreducible automorphisms of free groups, GAFA 7 (1997), 215-244.

3. 느, The Tits alternative for Out $\left(F_{n}\right)$, I: Dynamics of exponentially growing automorphisms, Ann. Math. 151 (2000), 517-623.

4. M. Bestvina and M. Handel, Train tracks and automorphisms of free groups, Ann. Math. 135 (1992), 1-51.

5. O. Bogopolski, Classification of automorphisms of the free group of rank 2 by ranks of fixedpoint subgroups, J. Group Theory 3 (2000), 339-351.

6. M. Bridson and D. Groves, Free-group automorphisms, train-tracks, and the beaded decomposition, arXiv:math.GR/0507589 v2, 2006.

7. Casson and Bleier, Automorphisms of surfaces after Nielsen and Thurston, London Mathematical Society Student Texts, vol. 9, Cambridge University Press, Cambridge, 1998.

8. D. Cooper, Automorphisms of free groups have finitely generated fixed point sets, J. Algebra 111 (1987), 453-456.

9. T. Coulbois, A. Hilion, and M. Lustig, $\mathbb{R}$-trees and laminations for free groups I: Algebraic laminations, preprint available on http://junon.u-3mrs.fr/hilion/, 2005.

10. J. L. Dyer and G. P. Scott, Periodic automorphisms of free groups, Comm. Alg. 3 (1975), 195-201.

11. A. Fathi, F. Laudenbach, and V. Poenaru (eds.), Travaux de Thurston sur les surfaces, Astérisque, vol. 66-67, Société Mathématique de France, Paris, 1976.

12. D. Gaboriau, A. Jaeger, G. Levitt, and M. Lustig, An index for counting fixed points of automorphisms of free groups, Duke Math. J. 93 (1998), 425-452.

13. A. Hilion and G. Levitt, Complexity of fixed points of automorphisms of free groups, in preparation, 2006.

14. N. Ivanov, Subgroups of Teichmüller modular groups, Translations of Mathematical Monographs, vol. 115, American Mathematical Society, Providence, 1992.

15. G. Levitt and M. Lustig, Most automorphisms of a hyperbolic group have very simple dynamics, Ann. Sci. ENS 33 (2000), 507-517.

16. _ Periodic ends, growth rates, Hölder dynamics for automorphisms of free groups, Comment. Math. Helv. 75 (2000), 415-429.

17. M. Lustig, Structure and conjugacy for automorphisms of free groups I, MPI-Preprint Series $130(2000)$.

18. J. McCool, Some finitely presented subgroups of the automorphism group of a free group, J. Algebra 35 (1975), 205-213.

19. J. Nielsen, Die Isomorphismen der allgemeinen, unendlichen Gruppe mit zwei Erzeugenden, Math. Ann. 78 (1917), 385-397.

20. U_ Untersuchungen zur Topologie der geschlossenen zweiseitigen Flächen, Acta Math. 50 (1927), 189-358, English translation in Collected Mathematical Papers, Birkhäuser (1986).

21. - Untersuchungen zur Topologie der geschlossenen zweiseitigen Flächen, II, Acta Math. 53 (1929), 1-76, English translation in Collected Mathematical Papers, Birkhäuser (1986).

22. __ Untersuchungen zur Topologie der geschlossenen zweiseitigen Flächen, III, Acta Math. 58 (1932), 87-167, English translation in Collected Mathematical Papers, Birkhäuser (1986).

23. W. Thurston, On the geometry and dynamics of diffeomorphisms of surfaces, Bull. AMS 19 (1988), 417-431.

LATP - Université Paul Cézanne Aix-Marseille III - Avenue de l'escadrille Normandie-Niémen, Case A - 13397 Marseille Cedex 20 - France

E-mail address: arnaud.hilion@univ-cezanne.fr 